\title{
Evaluation of 12 antibodies for distinguishing epithelioid mesothelioma from adenocarcinoma: identification of a three-antibody immunohistochemical panel with maximal sensitivity and specificity
}

\author{
Hadi Yaziji ${ }^{1}$, Hector Battifora ${ }^{2}$, Todd S Barry ${ }^{3}$, Harry C Hwang ${ }^{3}$, Carlos E Bacchi ${ }^{4}$, \\ Martin W McIntosh ${ }^{5}$, Steven J Kussick ${ }^{3}$ and Allen M Gown ${ }^{3}$ \\ ${ }^{1}$ Ancillary Pathways, Miami, FL, USA; ${ }^{2}$ Pathology Consultation Services, Arcadia, CA, USA; ${ }^{3}$ PhenoPath \\ Laboratories and Immunohistochemistry and Molecular Pathology Research Institute of Seattle, Seattle, \\ WA, USA; ${ }^{4}$ Consultoria em Patologia, Botucatu, Brazil and ${ }^{5} F r e d$ Hutchinson Cancer Research Center, \\ Seattle, WA, USA
}

\begin{abstract}
We evaluated the sensitivity and specificity of 10 monoclonal and two polyclonal antibodies for distinguishing epithelioid mesothelioma from adenocarcinoma (AdCA) using immunohistochemistry (IHC). The antibodies were directed against the mesothelial-associated antigens mesothelin, calretinin, cytokeratin 5 , thrombomodulin, Wilms' tumor-1 (WT-1) gene product and HBME-1, and the nonmesothelial antigens Lewis-Y blood group (antibody BG8), MOC-31, BerEp4, CD15, and carcinoembryonic antigen (CEA) family. The 133 tumors evaluated included 65 malignant epithelioid mesotheliomas, 22 lung AdCAs, 27 ovarian serous carcinomas, 24 breast carcinomas, and five gastric carcinomas. Diagnoses were based on clinical, histologic, ultrastructural, and/or IHC findings. Calretinin had the best sensitivity for mesothelioma (95\%), followed by HBME-1 (84\%), WT-1 (78\%), cytokeratin $5(76 \%)$, mesothelin $(75 \%)$, and vimentin and thrombomodulin $(68 \%)$. Thrombomodulin had the best specificity for mesothelioma (92\%), followed by cytokeratin $5(89 \%)$, calretinin $(87 \%)$ vimentin $(84 \%)$, and HBME$1(45 \%)$. When ovarian carcinomas were excluded from the analysis, the specificity of mesothelin and WT-1 for the diagnosis of mesothelioma increased to 90 and $81 \%$, respectively. The sensitivity of the nonmesothelial antigens for AdCA was organ dependent, with BG8 performing best in the breast cancer group (96\%), and BerEp4, BG8, MOC-31 performing best in the lung cancer group (100\%). The specificity of the nonmesothelial antigens for AdCA was $\mathbf{9 8 \%}$ for BG8 and CEA, $\mathbf{9 7 \%}$ for CD15, 95\% for BerEp4, and $\mathbf{8 7 \%}$ for MOC-31. A novel statistical analysis technique employing logic regression analysis identified a three-antibody immunohistochemical panel including calretinin, BG8, and MOC-31, which provided over $96 \%$ sensitivity and specificity for distinguishing epithelioid mesothelioma from AdCA.

Modern Pathology (2006) 19, 514-523. doi:10.1038/modpathol.3800534
\end{abstract}

Keywords: mesothelioma; immunohistochemistry; logic regression; pleura; asbestos; calretinin

The histologic distinction between malignant epithelioid mesothelioma (MEM) and adenocarcinoma (AdCA) is often difficult and requires ancillary studies. For many years, electron microscopy was the preferred ancillary method in this differential diagnosis. Typical ultrastructural features of MEM include long slender microvilli devoid of

Correspondence: Dr H Yaziji, MD, Ancillary Pathways, PO Box 43-0777, Miami, FL 33243-0777, USA.

E-mail: ancillarypath@mac.com

Received 28 June 2005; revised 28 September 2005; accepted 29 September 2005 gylocalyx over much of the cell membrane, and aberrant microvilli projecting through deficiencies in the basal lamina. ${ }^{1,2}$ However, these features are often absent in the less-differentiated MEM.

The development of antibodies to molecular markers of tissue differentiation has enabled immunohistochemistry (IHC) to supplant electron microscopy in distinguishing MEM from AdCA. Initially, antibodies to antigens commonly expressed by AdCA, such as carcinoembryonic antigen (CEA), LeuM1 (CD15), BerEp4, and B72.3 were used to distinguish MEM from AdCA. Thus, the diagnosis of MEM was based on the absence of expression of 
these proteins, an unsatisfying approach. More recently, several antigens expressed on mesothelial cells have been described. These proteins include calretinin, ${ }^{3,4}$ Wilms' tumor gene product (WT-1), ${ }^{5,6}$ mesothelin, ${ }^{7,8}$ cytokeratin 5,, ${ }^{9,10}$ HBME-1, ${ }^{11,12}$ and thrombomodulin. ${ }^{3,13}$

Calretinin, a calcium-binding protein widely expressed throughout the central and peripheral nervous systems, ${ }^{14}$ is also expressed by mesothelial cells and their tumors. Like the structurally related S-100 protein, calretinin is expressed in both the nucleus and cytoplasm. The earliest publications on calretinin reported low expression in MEM, but these studies employed a relatively insensitive antibody raised against guinea pig calretinin. ${ }^{11}$ Although Doglioni et $a l^{3}$ suggested that calretinin was mesothelium specific, subsequent studies have demonstrated its expression in a small but significant fraction of AdCAs. ${ }^{4,11}$

WT-1 is a DNA-binding protein predominantly located in the nucleus that plays a critical role in the development of the genitourinary tract. The Wilms' tumor gene is thought to represent a tumor suppressor gene; unlike more familiar tumor suppressor genes like p53, however, it appears to be operative only in selected cell types. In adult tissues, it is expressed by mesangial cells of the kidney, Sertoli cells of the testis, ovarian stromal cells and surface epithelium, mesothelial cells, and some smooth muscle and other stromal cells in the female genital tract (eg, myometrium, endometrial stromal cells). WT-1 is also expressed by MEM and, to a lesser extent, sarcomatoid mesothelioma, ${ }^{5,15}$ as well as tumors derived from the ovarian surface epithelium, which may be considered a modified mesothelium. Recent studies have confirmed the specificity of WT-1 for mesothelioma, ${ }^{16}$ but some studies have reported a relatively low sensitivity. ${ }^{17,18}$

Cytokeratin 5 is expressed by normal mesothelium, squamous and transitional epithelia, and myoepithelial cells., ${ }^{9,10,19-22}$ It is often expressed by MEM and squamous cell carcinoma, ${ }^{23}$ and can be an excellent marker for either one of these neoplasms. ${ }^{9,10}$ However, cytokeratin 5 is also expressed by a significant proportion of breast carcinomas. ${ }^{24}$

Mesothelin was originally known as the CAK1 antigen identified by the antibody $\mathrm{K} 1 .^{7,8,25,26}$ It is a $40 \mathrm{kD}$ cell surface protein that may be involved in cell-cell adhesion, and can now be identified by 'second generation' antimesothelin antibodies such as the 5B2 clone. This protein is highly specific for mesothelial cells and surface ovarian epithelial cells, and possibly pancreatic ductal carcinomas, ${ }^{27}$ and demonstrates a thick, linear, cell surface immunostaining pattern.

The following antigens are commonly expressed by AdCA, but not MEM: CEA family, ${ }^{28,29}$ CD15 (LeuM1) ${ }^{29-31}$ epithelial glycoprotein (B72.3) ${ }^{32,33}$ tumor glycoprotein (BerEp4), ${ }^{34,35}$ epithelial glycopro- tein (Bg8), ${ }^{11}$ E-cadherin, ${ }^{18}$ and tumor glycoprotein (MOC-31). ${ }^{17,36}$

The availability of antibodies to the abovementioned antigens has led to numerous publications with a wide spectrum of reported antibody performances. Given the large number of potential antigens one can evaluate to distinguish MEM from AdCA, there is clearly a need for critical evaluation of these antigens and their cognate antibodies, to establish a hierarchy of antigen/ antibody pairs in terms of sensitivity and specificity in the diagnosis of MEM and AdCA. Our study was designed to ascertain the sensitivity and specificity of each of these mesothelial markers and, using receiver operating characteristic (ROC) curve analysis, to identify an immunodiagnostic panel ideally suited for differentiating between MEM and AdCA.

\section{Materials and methods}

\section{IHC Methodology}

A total of 133 tumors were evaluated for the study, including 65 malignant epithelial mesotheliomas, 22 lung AdCAs, 27 ovarian serous carcinomas, 24 breast carcinomas, and five gastric carcinomas. Diagnoses had been made on clinical, histologic, ultrastructural, and/or IHC findings by one of the pathologists involved in this study. For each tumor, 4-6 $\mu \mathrm{m}$ sections were cut, and one hematoxylin- and eosin-stained section was prepared. Deparaffinized sections were immunostained with the 13 antibodies listed in Table 1. Of these, 12 antibodies were of interest for the study, while the 13th antibody (antipancytokeratin monoclonal antibody cocktail AE1/AE3) was used to confirm the antigenicity of the tissue. Note that these immunostains were performed in addition to any that had been performed to aid in the initial diagnosis of the case. The specific heat-induced epitope retrieval and detection methods (eg, avidin-biotin-peroxidase complex vs Envision $^{\mathrm{TM}}$ plus polymer) were selected based on prior optimization steps for each antibody. For details of the antibody clones, dilutions, and pretreatment see Table 1 .

The immunostained slides were simultaneously reviewed and scored by at least three of the pathologist participants and independently reviewed by all participants. Discrepancy in scoring was very infrequent ( $<2 \%$ of slides), and was resolved by a consensus review of all sections. A semiquantitative scoring system was used, reflecting the percentage of positive tumor cells to the nearest $10 \%$. For the sake of statistical analysis, the percentage cutoff for positive results was adjusted using several options including binary results (see below). Results for each antibody were tabulated from each of the 133 cases examined. 
Table 1 Antibodies used in this study

\begin{tabular}{lllll}
\hline Antibody & Clone & Source & Dilution & Pretreatment \\
\hline Calretinin & 5A5 & Novocastra & $1: 50$ & 8 min in pressure cooker, citrate buffer pH 6.0 \\
Mesothelin & $5 B 2$ & Novocastra & $1: 250$ & 8 min in pressure cooker, citrate buffer pH 6.0 \\
WT-1 & Polyclonal & Santa Cruz & $1: 1000$ & 8 min in pressure cooker, citrate buffer pH 6.0 \\
Anti-human mesothelial cell & HBME-1 & DAKO & $1: 250$ & 8 min in pressure cooker, citrate buffer pH 6.0 \\
Thrombomodulin & No09 & Novocastra & $1: 250$ & Steam $\times 20$ min in citrate+10 min in pronase \\
Cytokeratin 5/6 & D5/16B4 & Chemicon & $1: 2000$ & Steam $\times 20$ min in citrate+10 min in pronase \\
Vimentin & V9 & DAKO & $1: 1000$ & Steam $\times 20$ min in EDTA pH 8.0 \\
Epithelial glycoprotein & MOC-31 & DAKO & $1: 50$ & Steam $\times 20$ min in citrate pH 6.0 \\
Epithelial glycoprotein & BER-EP4 & DAKO & $1: 250$ & Protease XXIV $\times 5$ min \\
Epithelial glycoprotein (BG8) & F3 & Signet & $1: 250$ & Protease XXIV $\times 5$ min \\
CEA & Polyclonal & DAKO & $1: 4000$ & Steam $\times 20$ min in citrate pH 6.0 \\
CD15 & MMA & Becton Dickinson & $1: 50$ & Steam $\times 20$ min in citrate+10 min in pronase \\
Pan-cytokeratin (to confirm antigenicity) & AE1/AE3 & Chemicon & $1: 000$ & 8 min in pressure cooker, citrate buffer pH 6.0
\end{tabular}

\section{Statistical Analysis}

Initially, univariate analysis (Wilcoxon rank-sum test) was used to determine whether the individual antibodies could distinguish MEM from AdCA. ROC curves were then generated for each antibody. ROC curves are a graphical representation of the relationship between sensitivity and specificity over all possible diagnostic cutoff values. The area under the curve (AUC) in ROC analysis was used to compare the ability of an antibody to discriminate between mesothelioma and AdCA, allowing for visual identification of the antibodies with the greatest discriminatory power.

Multivariate statistical analysis was subsequently performed to identify the value of biomarker combinations. ${ }^{37}$ An adaptive binary regression method, logic regression (LR), ${ }^{38,39}$ was chosen over classical logistic regression because LR is more appropriate for combining binary predictor ${ }^{38-42} \mathrm{LR}$ methodology, which has been previously used for combining biomarkers ${ }^{41-44}$ produces 'and' and 'or' (Boolean) classification rules to identify combinations of variables best able to predict a certain outcome, and are easily interpreted by clinicians. In this study, we sought the combination of antibodies best suited to distinguish MEM from AdCA. Specifically, LR analysis was performed on a randomly selected series representing $50 \%$ of all tumors (the training data set). LR was then employed on the data from the remaining $50 \%$ of tumors (the validation data set), in an effort to confirm the results from the training set.

\section{Results}

As illustrated in Figure 1, the mesothelium-associated antigens demonstrated the following cellular localization patterns: nuclear (WT-1, Figure 1a); cytoplasmic (cytokeratin 5/6, Figure 1b and vimentin (not shown); combined nuclear and cytoplasmic (calretinin, Figure 1c); and membranous (meso- thelin, Figure 1d, thrombomodulin Figure 1e, and HBME-1, Figure 1f). The AdCA-associated antigens demonstrated the following cellular localization patterns (data not shown): membranous (BerEP4, CD15, MOC-31) and cytoplasmic (Bg8, polyclonal CEA).

Univariate statistical analysis using the Wilcoxon rank-sum significance test, a nonparametric statistical test, was performed to test the ability of each of the antibodies to distinguish MEM from AdCA (see Table 2, column 2). With the exception of the pan-cytokeratin cocktail AE1/AE3, which was only used to confirm that the tissues were antigenically intact, all 12 antibodies demonstrated a highly significant ability $(P<0.001)$ to distinguish MEM from AdCA, as indicated in the second column of Table 2. Further analysis of specific subtypes of AdCA was not undertaken because of the relatively small numbers of carcinomas of each organ type.

The relative ability of each antibody to distinguish MEM from AdCA was then formally determined by performing ROC curve analysis (see Figure 2). ROC curves demonstrate the coordinate variation in sensitivity (shown on the $Y$-axis) and specificity (1-specificity is shown on the $X$-axis) of a test as the threshold for defining test positivity varies over the entire range of possible test outcomes. Elevating the threshold will improve specificity but will sacrifice sensitivity, and vice versa. In this study, since antigen expression was determined to the nearest $10 \%$ of tumor cells positive, thresholds for defining positive antigen expression could vary from 0 to $100 \%$ in increments of $10 \%$. If one considers the mesothelin ROC curve as an example, this curve is flat along the horizontal axis until a false-positive rate of about $15 \%$ (1-specificity $=0.15$, at left part of curve), indicating that when the threshold of mesothelin positivity used to define MEM is set high, to allow very high specificity, this marker suffers from very low sensitivity. In contrast, when the threshold of mesothelin positivity used to define MEM is 

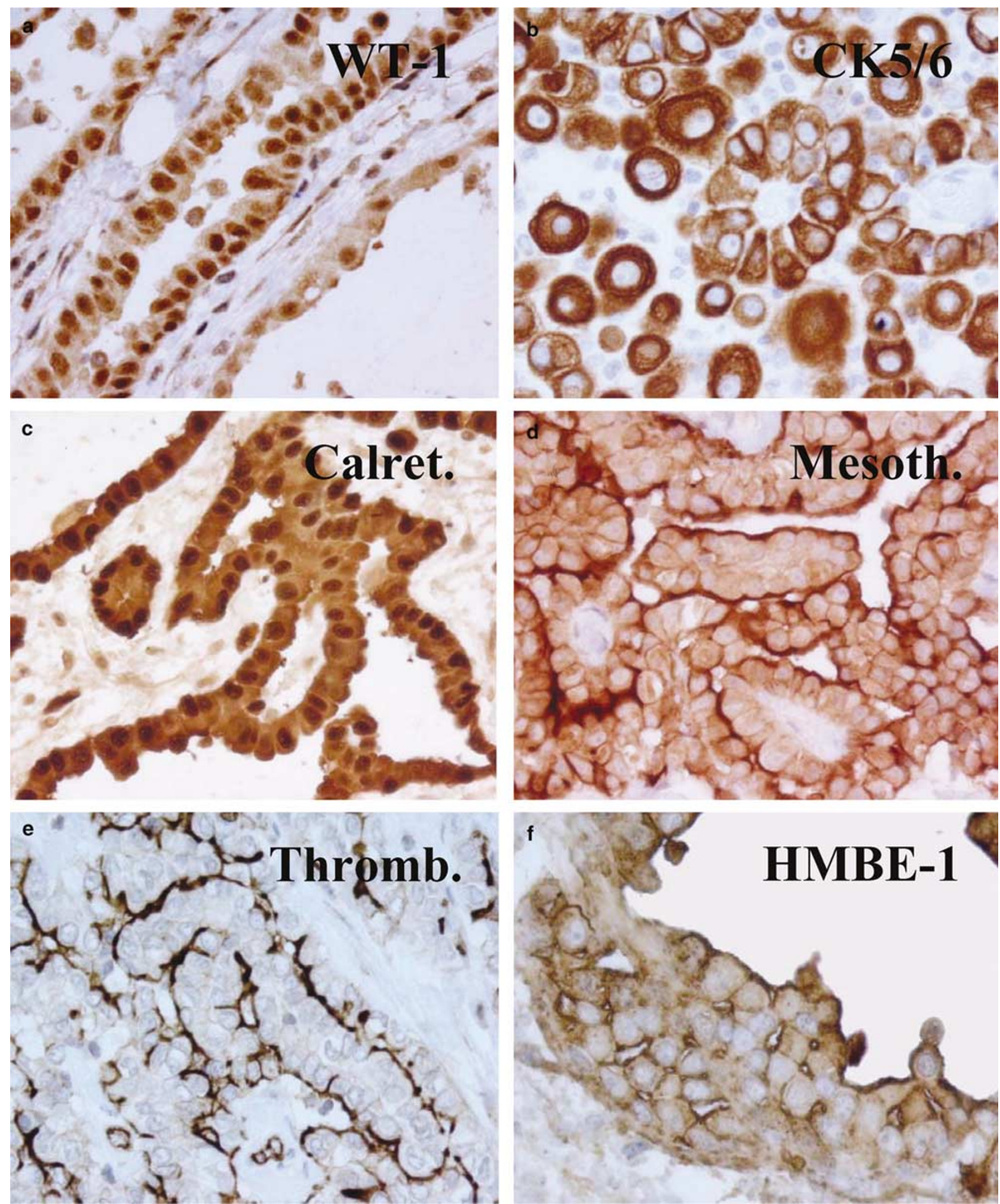

Figure 1 Summary of immunoreactivity patterns on MEMs. Immunohistochemical evaluation of a representative malignant epithelioid mesothelioma, demonstrating expression of the following antigens according to cellular localization pattern: nuclear (WT-1, a); cytoplasmic (cytokeratin 5/6, b); combined nuclear and cytoplasmic (calretinin, c); and membranous (mesothelin, d; thrombomodulin, e; and HBME-1, f).

lowered somewhat, the sensitivity first increases steeply with only a slight loss in specificity (steeply rising portion of curve), until sensitivity reaches a maximum. Further lowering the threshold for mesothelin fails to improve sensitivity, with a progressive decrease in specificity. 
The area under the ROC curve (or AUC) represents an optimal summary statistic for comparing the sensitivities and specificities of the 12 antibodies (see Table 2, column 3). The most intuitive way to interpret the AUC is to think of it as an average sensitivity of the marker (averaged over the possible specificities). Therefore, tests with a higher AUC are typically considered superior to tests with a lower AUC. A perfect test/marker has AUC of 1.0, and a noninformative test/marker has an AUC of 0.5. The AUC values are listed in the third column of Table 2, while the actual ROC curves appear in Figure 2. Note that calretinin had the greatest AUC, by far,

Table 2 Summary of discriminatory abilities of each marker in univariate analysis

\begin{tabular}{lcc}
\hline Marker & $\begin{array}{c}\text { Wilcoxon rank-sum } \\
\text { test (P-value) }\end{array}$ & $\begin{array}{c}\text { ROC curve analysis } \\
\text { (area } \text { under curve) }\end{array}$ \\
\hline Mesothelin & $<0.001$ & 0.613 \\
Calretinin & $<0.001$ & 0.906 \\
Ck5/6 & $<0.001$ & 0.722 \\
WT-1 & $<0.001$ & 0.637 \\
Thrombomodulin & $<0.001$ & 0.662 \\
HBME-1 & $<0.001$ & 0.679 \\
Vimentin & $<0.001$ & 0.622 \\
Bg8 & $<0.001$ & 0.956 \\
BerEP4 & $<0.001$ & 0.745 \\
CD15 & $<0.001$ & 0.531 \\
Polyclonal CEA & $<0.001$ & 0.631 \\
MOC-31 & $<0.001$ & 0.899 \\
\end{tabular}
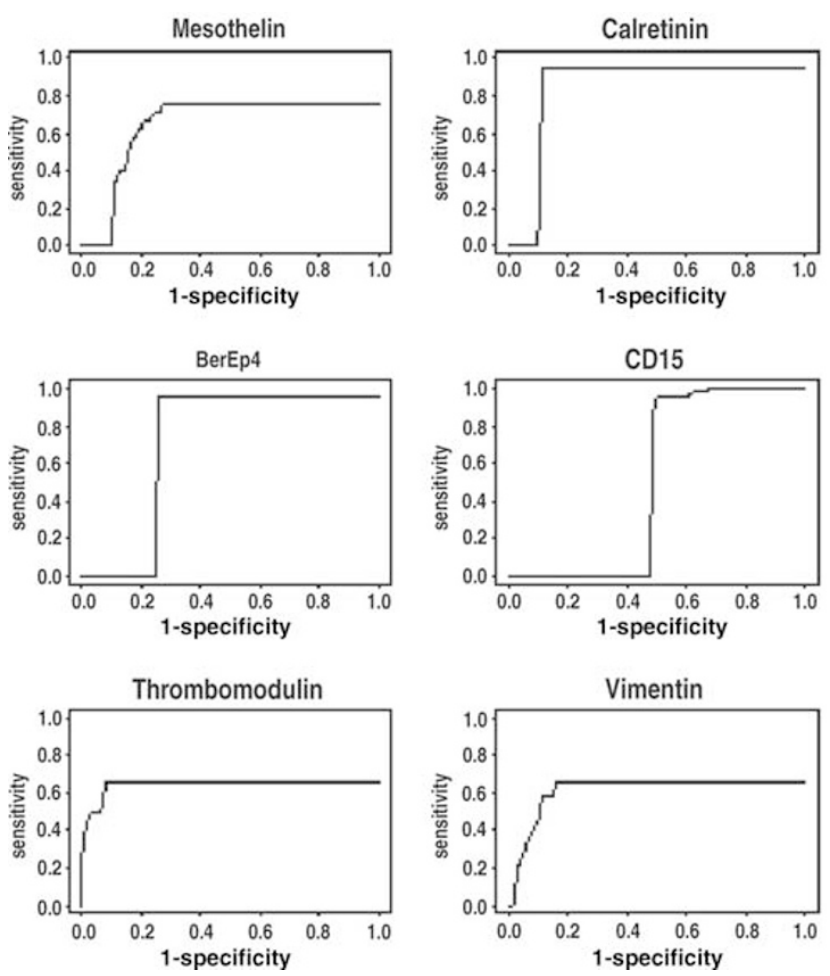

among the MEM-associated antibodies, while Bg8 and MOC-31 clearly had the greatest AUCs among the AdCA-associated antibodies.

In Table 3a, the sensitivities and specificities for each of the MEM-associated antibodies was determined for the diagnosis of MEM, while in Table $3 \mathrm{~b}$ the sensitivities and specificities for each of the AdCA-associated antibodies was determined for the diagnosis of AdCA. In both cases, using a fixed positive cutoff of $10 \%$ antigen-positive tumor cells

Table 3 Sensitivity of specificity of each marker, using a fixed $10 \%$ positive cutoff

\begin{tabular}{lccc}
\hline $\begin{array}{l}\text { For Dx. of } \\
\text { mesothelioma }\end{array}$ & Sensitivity & Specificity & $\begin{array}{c}\text { Specificity } \\
\text { excluding } \\
\text { ovarian tumors }\end{array}$ \\
\hline Mesothelin & 0.75 & 0.71 & 0.9 \\
WT-1 & 0.78 & 0.62 & 0.81 \\
Calretinin & 0.95 & 0.87 & \\
Cytokeratin 5/6 & 0.76 & 0.89 & \\
Thrombomodulin & 0.68 & 0.92 & \\
HBME-1 & 0.84 & 0.48 & \\
Vimentin & 0.69 & 0.84 & \\
& & & \\
For Dx. of & & & \\
adenocarcinoma & & & \\
BG8 & 0.95 & 0.98 & \\
BerEP4 & 0.74 & 0.95 & \\
CD15 & 0.51 & 0.97 & \\
CEA & 0.63 & 0.98 & \\
MOC-31 & 0.92 & 0.87 & \\
& & &
\end{tabular}
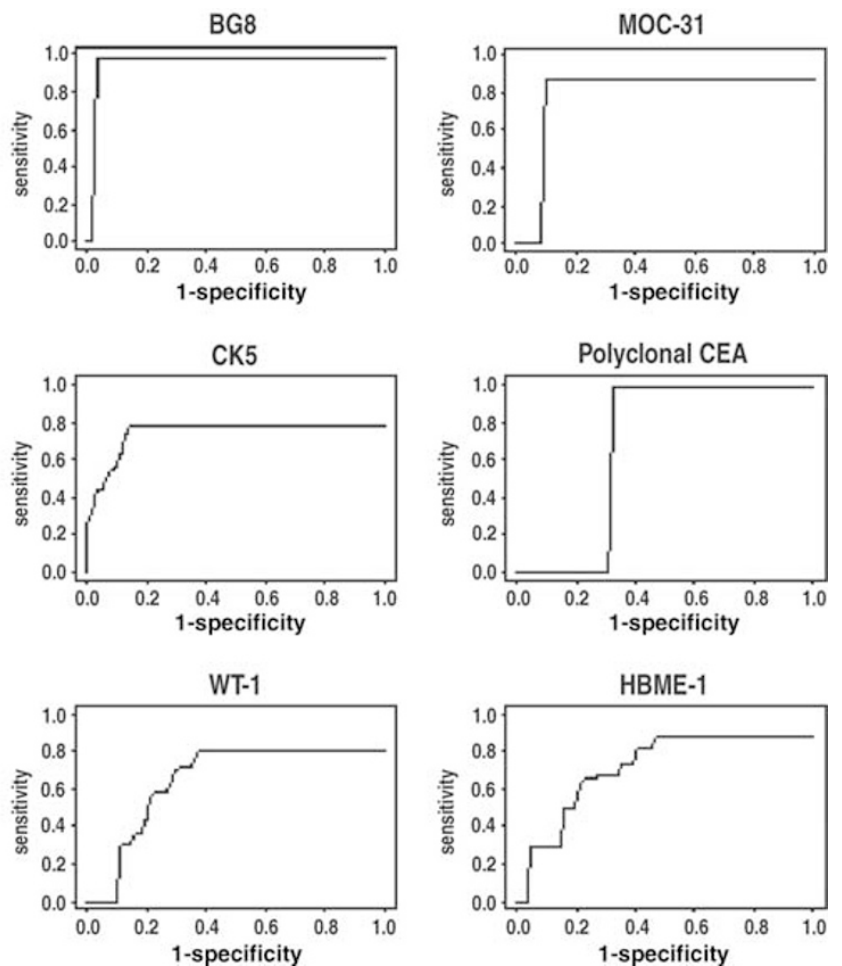

Figure 2 ROC curve analysis for each of the 12 antibodies evaluated. 


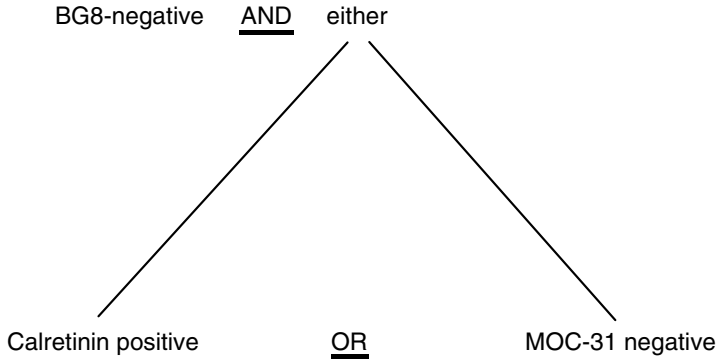

Figure 3 Logic tree for discriminating MEM from AdCA. This approach correctly identified 63 of 65 MEMs, with no falsepositive MEMs identified.

was used. The $10 \%$ positive cutoff was chosen because only a minimal number of the specimens in this study showed immunoreactivity on $0-10 \%$ of the tumor cells. Strikingly, the antibody to calretinin was over $90 \%$ sensitive for the identification of MEM vs AdCA, while the antibody to thrombomodulin was over $90 \%$ specific for the diagnosis of MEM vs AdCA (see Table 3). Not surprisingly, the specificity of the mesothelin and WT-1 antibodies for mesothelioma could be increased about $30 \%$ if ovarian serous carcinomas, which coexpress mesothelin and WT-1, were excluded from the analysis. In contrast to the MEM data, the antibodies to BG8 and MOC-31 were over 90\% sensitive for the identification of AdCA vs MEM, while the antibodies to BG8, BerEP4, CD15, and CEA were all over $90 \%$ specific for the diagnosis of AdCA vs MEM.

Logic (not logistic) regression (LR), which uses Boolean 'and' and 'or' rules to develop a model for predicting an outcome of interest, was applied to determine the optimal combination of antibodies to distinguish carcinomas and mesotheliomas with few errors. In this analysis, 'or' rules improve sensitivity and 'and' rules improve specificity. Immunoreactivity for each antibody was treated as a dichotomous variable (positive vs negative depending on whether or not $10 \%$ of the tumor cells expressed the marker of interest). It has been shown that in such settings the general principle of using binary regression methods to combine markers is optimal, ${ }^{37}$ and logistic regression is a binary approach suitable for dichotomous variables. ${ }^{40,42}$

LR analysis was first applied to the training data set of $50 \%$ of the cases. This analysis demonstrated that the number of antibodies required for optimal discrimination of MEM from AdCA could be reduced to three-BG8, calretinin, and MOC31without decreasing the sensitivity or specificity to less than 96\% (only one mesothelioma case was incorrectly classified as AdCA). Specifically, the combination of BG8 negativity with either calretinin positivity or MOC31 negativity demonstrated a sensitivity of $100 \%$ and specificity of $96 \%$ in confirming the diagnosis of mesothelioma (see Figure 3 for a schematic depiction of an algorithm for distinguishing MEM from AdCA based on this data). Both the training data set and the validation data set each failed to identify only one tumor as mesothelioma.

\section{Discussion}

Various tumors with epithelioid histology can affect the peripheral lung, pleura, and chest wall. These include epithelioid mesotheliomas, AdCAs, epithelioid nerve sheath tumors, melanomas, and pseudomesotheliomatous angiosarcomas. ${ }^{45}$ By far, the most common tumors in this category are AdCA and mesothelioma. Therefore, our study focused on the most common differential diagnostic issue in this setting, namely, the distinction between epithelioid mesothelioma and AdCA involving serous surfaces.

There have been some excellent reviews of the immunohistochemical approaches to this problem $^{1,11,12,46-52}$ The importance of using a panel of markers rather than a single one was well recognized in the early years of IHC. ${ }^{53-55}$ Recent studies identified some antibodies with acceptable rates of specificity and sensitivity in distinguishing MEM from $\mathrm{AdCA}^{3,4,10,16,22,35,56-59}$ although other antibodies have yielded suboptimal results. ${ }^{60-63}$

Two major strengths of our study were the utilization of a broad panel of antibodies to the most common markers of mesotheliomas and AdCAs, and the large number of cases evaluated. Selection of the specific antibodies was based on both evaluation of the literature ${ }^{17,48,64-68}$ and on clinical experience in our laboratories. A few potentially useful markers, such as E-cadherin and $\mathrm{N}$-cadherin, were omitted because of conflicting reports about their performance in distinguishing mesothelioma from AdCA. ${ }^{57,69-72}$

A third strength of our study was the detailed statistical analysis, which distinguishes this study from most previous studies in this area. Not only were all antibodies evaluated by univariate analysis to determine their abilities to distinguish MEM from all AdCA (Table 2, column 2), but the relative utility of each antibody was quantified by ROC curve analysis (Table 2, column 3), the relevant sensitivities and specificities were calculated for each MEM- and AdCA-associated antibody (Table 3), and LR was applied to determine the optimal combination of antibodies for distinguishing MEM from AdCA.

The quality of a marker is determined by the extent to which sensitivity and specificity both remain high at the threshold set to define marker positivity. As shown in Figure 2, the AUC is a convenient measurement for comparing the 
overall quality of markers. Calretinin is clearly the best marker for positively identifying MEM, in that it has the highest sensitivity, with relatively high specificity; HBME-1 achieves over $80 \%$ sensitivity in identifying MEM, but with a specificity of about $50 \%$. While BerEP4, BG8, CD15, polyclonal CEA, and MOC-31 all are capable of identifying AdCA with high sensitivity, concurrent high specificity clearly makes BG8 and MOC-31 the optimal markers for positively identifying AdCA.

Importantly, the LR model identified three antibodies (BG8, calretinin, and MOC-31) capable of distinguishing MEM from AdCA with 100\% specificity and $96 \%$ sensitivity when this model was applied to the validation data set. Since LR analysis was so effective at identifying optimal markers for distinguishing MEM from AdCA, this is likely to represent a general method for optimizing antibody panels to distinguish between other histologically similar entities.

Despite the power of LR as an analytical technique, it is critical to remember that the quality of any immunohistochemical panel to distinguish MEM from AdCA will be influenced by the choice of antigens evaluated, the choice of antibody clones, the type of tissue, and the gender of the patient. While the influence of the type of tissue (eg, surgical biopsy material vs cell block of cytological material vs autopsy material) on the quality of immunohistochemical data is obvious, the importance of antigen and antibody choice is exemplified by a recent study ${ }^{63}$ in which an anticalretinin antibody with relatively poor specificity was employed, and antibodies to sensitive and specific mesothelial-restricted proteins (eg, mesothelin, cytokeratin 5, WT-1) were not employed. Patient gender directly impacts the specificity of both mesothelin and WT-1 to distinguish MEM from AdCA, since both antigens are expressed by ovarian serous carcinomas. Therefore, we advocate the use of 'gender-neutral' panel, which includes calretinin instead of WT-1 and mesothelin. The combination of calretinin, BG8, and MOC-31 would seem to be applicable across most clinicopathologic scenarios.

There are several caveats regarding these findings. First, although tumor cells in fluids typically maintain their immunophenotypes, we did not confirm our classification algorithm in malignant pleural fluid effusions. Second, we did not confirm our classification algorithm among peritoneal mesotheliomas, although such mesotheliomas appear to have slightly different characteristics than pleural mesotheliomas. ${ }^{73}$ Third, we did not study metastatic carcinomas to the pleura, but focused on primary tumors of the respective organs (lung, ovary, breast, stomach). While immunophenotypic fidelity of metastatic tumors compared to their origins has been documented, ${ }^{74}$ it is possible that a particular antigen expressed at high level by a primary tumor could be lost or significantly reduced in the metastatic setting. Fourth, we did not specifically address the differential diagnosis of MEM vs other types of AdCA, including prostate, pancreatic, and renal cell carcinomas, although the latter issue was recently addressed. ${ }^{75}$ Fifth, we did not evaluate monoclonal antibodies to D2-40 and podoplanin, which also appear to be highly sensitive for identifying MEM. ${ }^{76-78}$ Note that D2-40, like WT-1 and mesothelin, also recognized ovarian serous carcinomas in one of these two studies, ${ }^{76}$ and therefore does not appear to be highly specific for MEM. The two papers describing podoplanin in MEM and $\operatorname{AdCA}^{77,78}$ suggest that it is highly specific for MEM. We are uncertain whether adding either D2-40 or podoplanin to the combination of BG8, calretinin, and MOC-31 will improve the ability to discriminate MEM from AdCA.

In conclusion, our study goes beyond most previous publications in this area, in the following ways: (a) we evaluated a particularly large number of markers, for which the immunohistochemical techniques were previously screened and optimized; (b) we evaluated a very large set of MEM and AdCA from numerous geographic locations; and (c) we used a variety of robust statistical methods ${ }^{38-42}$ to design a cost-effective panel of only three monoclonal antibodies, without compromising sensitivity or specificity.

\section{References}

1 Leong AS, Stevens MW, Mukherjee TM. Malignant mesothelioma: cytologic diagnosis with histologic, immunohistochemical, and ultrastructural correlation. Semin Diagn Pathol 1992;9:141-150.

2 Di Muzio M, Spoletini L, Strizzi L, et al. Prognostic significance of presence and reduplication of basal lamina in malignant pleural mesothelioma. Hum Pathol 2000;31:1341-1345.

3 Doglioni C, Tos AP, Laurino L, et al. Calretinin: a novel immunocytochemical marker for mesothelioma. Am J Surg Pathol 1996;20:1037-1046.

4 Ordonez NG. Value of calretinin immunostaining in differentiating epithelial mesothelioma from lung adenocarcinoma. Mod Pathol 1998;11:929-933.

5 Amin KM, Litzky LA, Smythe WR, et al. Wilms' tumor 1 susceptibility (WT1) gene products are selectively expressed in malignant mesothelioma. Am J Pathol 1995;146:344-356.

6 Kumar-Singh S, Segers K, Rodeck U, et al. WT1 mutation in malignant mesothelioma and WT1 immunoreactivity in relation to p53 and growth factor receptor expression, cell-type transition, and prognosis. J Pathol 1997;181:67-74.

7 Chang K, Pai LH, Pass H, et al. Monoclonal antibody $\mathrm{K} 1$ reacts with epithelial mesothelioma but not with lung adenocarcinoma. Am J Surg Pathol 1992;16: 259-268.

8 Chang K, Pastan I. Molecular cloning of mesothelin, a differentiation antigen present on mesothelium, mesotheliomas, and ovarian cancers. Proc Natl Acad Sci USA 1996;93:136-140. 
9 Clover J, Oates J, Edwards C. Anti-cytokeratin 5/6: a positive marker for epithelioid mesothelioma. Histopathology 1997;31:140-143.

10 Ordonez N. Value of cytokeratin 5/6 immunostaining in distinguishing epithelial mesothelioma of the pleura from lung adenocarcinoma. Am J Surg Pathol 1998;22:1215-1221.

11 Riera JR, Astengo-Osuna C, Longmate JA, et al. The immunohistochemical diagnostic panel for epithelial mesothelioma: a reevaluation after heat-induced epitope retrieval [see comments]. Am J Surg Pathol 1997; 21:1409-1419.

12 Ordonez NG. The value of antibodies 44-3A6, SM3, HBME-1, and thrombomodulin in differentiating epithelial pleural mesothelioma from lung adenocarcinoma: a comparative study with other commonly used antibodies. Am J Surg Pathol 1997;21:13991408.

13 Collins CL, Ordonez NG, Schaefer R, et al. Thrombomodulin expression in malignant pleural mesothelioma and pulmonary adenocarcinoma. Am J Pathol 1992;141:827-833.

14 Andressen C, Blumcke I, Celio MR. Calcium-binding proteins: selective markers of nerve cells. Cell Tissue Res 1993;271:181-208.

15 Walker C, Rutten F, Yuan X, et al. Wilms' tumor suppressor gene expression in rat and human mesothelioma. Cancer Res 1994;54:3101-3106.

16 Hecht JL, Lee BH, Pinkus JL, et al. The value of Wilms tumor susceptibility gene 1 in cytologic preparations as a marker for malignant mesothelioma. Cancer 2002; 96:105-109.

17 Oates J, Edwards C. HBME-1, MOC-31, WT1 and calretinin: an assessment of recently described markers for mesothelioma and adenocarcinoma. Histopathology 2000;36:341-347.

18 Ordonez NG. Value of thyroid transcription factor-1, E-cadherin, BG8, WT1, and CD44S immunostaining in distinguishing epithelial pleural mesothelioma from pulmonary and nonpulmonary adenocarcinoma. Am J Surg Pathol 2000;24:598-606.

19 Nagle RB, Bocker W, Davis JR, et al. Characterization of breast carcinomas by two monoclonal antibodies distinguishing myoepithelial from luminal epithelial cells. J Histochem Cytochem 1986;34:869-881.

20 Cury PM, Butcher DN, Fisher C, et al. Value of the mesothelium-associated antibodies thrombomodulin, cytokeratin 5/6, calretinin, and CD44H in distinguishing epithelioid pleural mesothelioma from adenocarcinoma metastatic to the pleura. Mod Pathol 2000; 13:107-112.

21 Chu PG, Weiss LM. Expression of cytokeratin 5/6 in epithelial neoplasms: an immunohistochemical study of 509 cases. Mod Pathol 2002;15:6-10.

22 Attanoos RL, Webb R, Dojcinov SD, et al. Value of mesothelial and epithelial antibodies in distinguishing diffuse peritoneal mesothelioma in females from serous papillary carcinoma of the ovary and peritoneum. Histopathology 2002;40:237-244.

23 Moll R, Dhouailly D, Sun TT. Expression of keratin 5 as a distinctive feature of epithelial and biphasic mesotheliomas. An immunohistochemical study using monoclonal antibody AE14. Virchows Arch B Cell Pathol Incl Mol Pathol 1989;58:129-145.

24 Kaufmann O, Fietze E, Mengs J, et al. Value of p63 and cytokeratin 5/6 as immunohistochemical markers for the differential diagnosis of poorly differentiated and undifferentiated carcinomas. Am J Clin Pathol 2001; 116:823-830.

25 Chang K, Pastan I, Willingham MC. Isolation and characterization of a monoclonal antibody, $\mathrm{K} 1$, reactive with ovarian cancers and normal mesothelium. Int J Cancer 1992;50:373-381.

26 Chang K, Pai LH, Batra JK, et al. Characterization of the antigen (CAK1) recognized by monoclonal antibody K1 present on ovarian cancers and normal mesothelium. Cancer Res 1992;52:181-186.

27 Argani P, Iacobuzio-Donahue C, Ryu B, et al. Mesothelin is overexpressed in the vast majority of ductal adenocarcinomas of the pancreas: identification of a new pancreatic cancer marker by serial analysis of gene expression (SAGE). Clin Cancer Res 2001;7: 3862-3868.

28 Battifora H, Kopinski MI. Distinction of mesothelioma from adenocarcinoma. An immunohistochemical approach. Cancer 1985;55:1679-1685.

29 Wirth PR, Legier J, Wright Jr GL. Immunohistochemical evaluation of seven monoclonal antibodies for differentiation of pleural mesothelioma from lung adenocarcinoma. Cancer 1991;67:655-662.

30 Sheibani K, Battifora H, Burke JS. Antigenic phenotype of malignant mesotheliomas and pulmonary adenocarcinomas. An immunohistologic analysis demonstrating the value of Leu M1 antigen. Am J Pathol 1986; 123:212-219.

31 Sheibani K, Battifora H, Burke JS, et al. Leu-M1 antigen in human neoplasms. An immunohistologic study of 400 cases. Am J Surg Pathol 1986;10:227-236.

32 Ordonez NG. The immunohistochemical diagnosis of mesothelioma. Differentiation of mesothelioma and lung adenocarcinoma. Am J Surg Pathol 1989;13: 276-291.

33 Wick MR, Loy T, Mills SE, et al. Malignant epithelioid pleural mesothelioma versus peripheral pulmonary adenocarcinoma: a histochemical, ultrastructural, and immunohistologic study of 103 cases. Hum Pathol 1990;21:759-766.

34 Gaffey MJ, Mills SE, Swanson PE, et al. Immunoreactivity for BER-EP4 in adenocarcinomas, adenomatoid tumors, and malignant mesotheliomas. Am J Surg Pathol 1992;16:593-599.

35 Ordonez NG. Value of the Ber-EP4 antibody in differentiating epithelial pleural mesothelioma from adenocarcinoma. The MD Anderson experience and a critical review of the literature. Am J Clin Pathol 1998;109:85-89.

36 Ordonez NG. Value of the MOC-31 monoclonal antibody in differentiating epithelial pleural mesothelioma from lung adenocarcinoma. Hum Pathol 1998;29: 166-169.

37 McIntosh MW, Pepe MS. Combining several screening tests: optimality of the risk score. Biometrics 2002;58: $657-664$

38 Smekal G, Scharl A, von Duvillard SP, et al. Accuracy of neuro-fuzzy logic and regression calculations in determining maximal lactate steady-state power output from incremental tests in humans. Eur J Appl Physiol 2002;88:264-274.

39 Kooperberg C, Ruczinski I. Identifying interacting SNPs using Monte Carlo logic regression. Genet Epidemiol 2005;28:157-170.

40 Kooperberg C, Ruczinski I, LeBlanc ML, et al. Sequence analysis using logic regression. Genet Epidemiol 2001;21(Suppl 1):S626-S631. 
41 Keles S, van der Laan MJ, Vulpe C. Regulatory motif finding by logic regression. Bioinformatics 2004;20: 2799-2811.

42 Janes H, Pepe M, Kooperberg C, et al. Identifying target populations for screening or not screening using logic regression. Stat Med 2005;24:13211338.

43 Etzioni R, Falcon S, Gann PH, et al. Prostate-specific antigen and free prostate-specific antigen in the early detection of prostate cancer: do combination tests improve detection? Cancer Epidemiol Biomarkers Prev 2004;13:1640-1645.

44 Feng Q, Balasubramanian A, Hawes SE, et al. Detection of hypermethylated genes in women with and without cervical neoplasia. J Natl Cancer Inst 2005; 97:273-282.

45 Falconieri G, Bussani R, Mirra M, et al. Pseudomesotheliomatous angiosarcoma: a pleuropulmonary lesion simulating malignant pleural mesothelioma. Histopathology 1997;30:419-424.

46 Ordonez NG. The immunohistochemical diagnosis of epithelial mesothelioma. Hum Pathol 1999;30 313-323.

47 Ordonez NG. In search of a positive immunohistochemical marker for mesothelioma: an update. Adv Anat Pathol 1998;5:53-60.

48 Moran CA, Wick MR, Suster S. The role of immunohistochemistry in the diagnosis of malignant mesothelioma. Semin Diagn Pathol 2000;17:178-183.

49 Sheibani K. Immunopathology of malignant mesothelioma. Hum Pathol 1994;25:219-220.

50 Leong AS, Vernon-Roberts E. The immunohistochemistry of malignant mesothelioma. Pathol Annu 1994; 29(Part 2):157-179.

51 Bedrossian CW, Bonsib S, Moran C. Differential diagnosis between mesothelioma and adenocarcinoma: a multimodal approach based on ultrastructure and immunocytochemistry. Semin Diagn Pathol 1992; 9:124-140.

52 Sheibani K, Esteban JM, Bailey A, et al. Immunopathologic and molecular studies as an aid to the diagnosis of malignant mesothelioma. Hum Pathol 1992;23: 107-116.

53 Otis CN, Carter D, Cole S, et al. Immunohistochemical evaluation of pleural mesothelioma and pulmonary adenocarcinoma. A bi-institutional study of 47 cases. Am J Surg Pathol 1987;11:445-456.

54 Corson JM, Pinkus GS. Mesothelioma: profile of keratin proteins and carcinoembryonic antigen: an immunoperoxidase study of 20 cases and comparison with pulmonary adenocarcinomas. Am J Pathol 1982; 108:80-87.

55 Churg A. Immunohistochemical staining for vimentin and keratin in malignant mesothelioma. Am J Surg Pathol 1985;9:360-365.

56 Sosolik RC, McGaughy VR, De Young BR. Anti-MOC31: a potential addition to the pulmonary adenocarcinoma versus mesothelioma immunohistochemistry panel. Mod Pathol 1997;10:716-719.

57 Leers MP, Aarts MM, Theunissen PH. E-cadherin and calretinin: a useful combination of immunochemical markers for differentiation between mesothelioma and metastatic adenocarcinoma. Histopathology 1998;32: 209-216.

58 Brockstedt U, Gulyas M, Dobra K, et al. An optimized battery of eight antibodies that can distinguish most cases of epithelial mesothelioma from adenocarcinoma. Am J Clin Pathol 2000;114: 203-209.

59 Carella R, Deleonardi G, D’Errico A, et al. Immunohistochemical panels for differentiating epithelial malignant mesothelioma from lung adenocarcinoma: a study with logistic regression analysis. Am J Surg Pathol 2001;25:43-50.

60 Betta PG. The role of immunohistochemistry in the diagnosis of malignant mesothelioma. Monaldi Arch Chest Dis 1998;53:186-187.

61 Dejmek A, Hjerpe A. Reactivity of six antibodies in effusions of mesothelioma, adenocarcinoma and mesotheliosis: stepwise logistic regression analysis. Cytopathology 2000;11:8-17.

62 Chenard-Neu MP, Kabou A, Mechine A, et al. Immunohistochemistry in the differential diagnosis of mesothelioma and adenocarcinoma. Evaluation of 5 new antibodies and 6 traditional antibodies. Ann Pathol 1998;18:460-465.

63 Roberts F, Harper CM, Downie I, et al. Immunohistochemical analysis still has a limited role in the diagnosis of malignant mesothelioma. A study of thirteen antibodies. Am J Clin Pathol 2001;116: 253-262.

64 Osborn M, Pelling N, Walker MM, et al. The value of 'mesothelium-associated' antibodies in distinguishing between metastatic renal cell carcinomas and mesotheliomas. Histopathology 2002;41: 301-307.

65 Abutaily AS, Addis BJ, Roche WR. Immunohistochemistry in the distinction between malignant mesothelioma and pulmonary adenocarcinoma: a critical evaluation of new antibodies. J Clin Pathol 2002;55: 662-668.

66 Wick MR, Moran CA, Mills SE, et al. Immunohistochemical differential diagnosis of pleural effusions, with emphasis on malignant mesothelioma. Curr Opin Pulm Med 2001;7:187-192.

67 Ordonez NG. Role of immunohistochemistry in differentiating epithelial mesothelioma from adenocarcinoma. Review and update. Am J Clin Pathol 1999;112: 75-89.

68 Comin CE, Novelli L, Boddi V, et al. Calretinin, thrombomodulin, CEA, and CD15: a useful combination of immunohistochemical markers for differentiating pleural epithelial mesothelioma from peripheral pulmonary adenocarcinoma. Hum Pathol 2001;32: 529-536.

69 Peralta S-A, Knudsen KA, Jaurand MC, et al. The differential expression of $\mathrm{N}$-cadherin and E-cadherin distinguishes pleural mesotheliomas from lung adenocarcinomas [see comments]. Hum Pathol 1995;26: 1363-1369.

70 Kitazume H, Kitamura K, Mukai K, et al. Cytologic differential diagnosis among reactive mesothelial cells, malignant mesothelioma, and adenocarcinoma: utility of combined E-cadherin and calretinin immunostaining. Cancer 2000;90:55-60.

71 Ordonez NG. The immunohistochemical diagnosis of mesothelioma: a comparative study of epithelioid mesothelioma and lung adenocarcinoma. Am J Surg Pathol 2003;27:1031-1051.

72 Ordonez NG. Value of E-cadherin and N-cadherin immunostaining in the diagnosis of mesothelioma. Hum Pathol 2003;34:749-755.

73 Trupiano JK, Geisinger KR, Willingham MC, et al. Diffuse malignant mesothelioma of the peritoneum 
and pleura, analysis of markers. Mod Pathol 2004;17: 476-481.

74 Esteban JM, Battifora H. Tumor immunophenotype: comparison between primary neoplasm and its metastases. Mod Pathol 1990;3:192-197.

75 Ordonez NG. The diagnostic utility of immunohistochemistry in distinguishing between mesothelioma and renal cell carcinoma: a comparative study. Hum Pathol 2004;35:697-710.
76 Chu AY, Litzky LA, Pasha TL, et al. Utility of D2-40, a novel mesothelial marker, in the diagnosis of malignant mesothelioma. Mod Pathol 2005;18:105-110.

77 Kimura N, Kimura I. Podoplanin as a marker for mesothelioma. Pathol Int 2005;55:83-86.

78 Ordonez NG. D2-40 and podoplanin are highly specific and sensitive immunohistochemical markers of epithelioid malignant mesothelioma. Hum Pathol 2005;36: 372-380. 